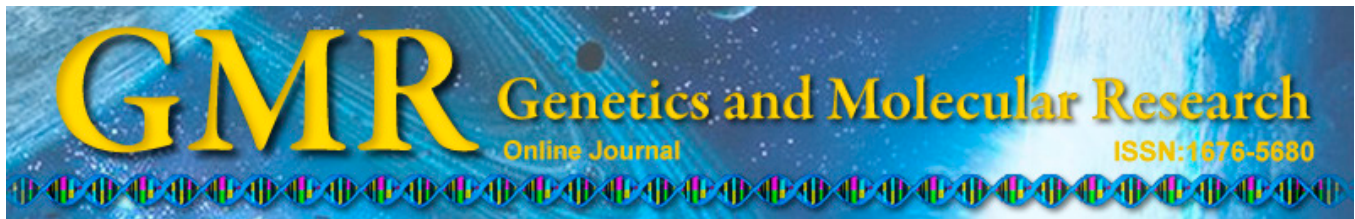

\title{
Next-generation sequencing identification and characterization of microsatellite markers in Aconitum austrokoreense Koidz., an endemic and endangered medicinal plant of Korea
}

\author{
Y.-E. Yun, J.-N. Yu, G.H. Nam, S.-A. Ryu, S. Kim, K. Oh and C.E. Lim \\ National Institute of Biological Resources, Environmental Research Complex, \\ Incheon, Korea \\ Corresponding author: C.E. Lim \\ E-mail: Chaelim@korea.kr
}

Genet. Mol. Res. 14 (2): 4812-4817 (2015)

Received June 11, 2014

Accepted October 29, 2014

Published May 11, 2015

DOI http://dx.doi.org/10.4238/2015.May.11.13

\begin{abstract}
We used next-generation sequencing to develop 9 novel microsatellite markers in Aconitum austrokoreense, an endemic and endangered medicinal plant in Korea. Owing to its very limited distribution, over-harvesting for traditional medicinal purposes, and habitat loss, the natural populations are dramatically declining in Korea. All novel microsatellite markers were successfully genotyped using 64 samples from two populations (Mt. Choejeong, Gyeongsangbukdo and Ungseokbong, Gyeongsangnam-do) of Gyeongsang Province. The number of alleles ranged from 2 to 7 per locus in each population. Observed and expected heterozygosities ranged from 0.031 to 0.938 and from 0.031 to 0.697 , respectively. The novel markers will be valuable tools for assessing the genetic diversity of $A$. austrokoreense and for germplasm conservation of this endangered species.
\end{abstract}

Key words: Aconitum austrokoreense; Microsatellite marker; Endemic and endangered medicinal plant, Next-generation sequencing; Genetic diversity 


\section{INTRODUCTION}

Aconitum austrokoreense Koidz. belongs to the subgenus Aconitum sect. Flagellaria (Steinb.) Nakai ser. Racemulosa W. T. Wang, and differs from other species in the series by having undivided or indistinct 3-lobed leaves. The Racemulosa series consists of three species, with the other two species endemic to South Western and Central China and preferentially growing in rocky habitats. In contrast, A. austrokoreense is restricted to the Southeastern part (mainly Gyeongsang Province) of the Korean peninsula.

Since ancient times, Aconitum species have been well known in Eastern Asia for their toxicity and as potent herbal medicines (Lee, 1979; Bisset, 1981; Bessonova et al., 1987; Nautiyal et al., 2009; Liang., 2012; Tang et al., 2012). The dried root of Aconitum species (common names: "Buja" in Korea, "Chuanwu" in China, and "Vatsanabha" in India) is a widely used drug in traditional medical practices all over the world (Lyu et al., 2008; He et al., 2010; Rastogi, 2011). In particular, Aconitum austrokoreense is commonly used for medicinal purposes in Gyeongsang Province (Lim C.E. \& Lee H.-W. personal communication). The species shows a very limited geographic distribution, with only a few isolated populations in rocky habitats near the trails along the mountain valleys of Gyeongsang Province in Korea (Kadota, 1987; Park, 2007). Because of its limited distribution, over-harvesting for traditional medicinal use together with habitat loss have resulted in a dramatic decline of the natural Aconitum populations (Suh et al., 2004; Yoo et al., 2005). Thus, A. austrokoreense was listed in the Red Data Book of Endangered Vascular Plants in Korea (Suh and Kim, 2012).

In general, subgenus Aconitum species are known to have bisexual, protandrous flowers and propagate by both seeds and daughter tubers (Kadota, 1987; Brink, 1980; Oh and Park, 1998; Bosch and Waser, 1999; Chung and Park, 2000). However, some species have seeds with very low germination rates and reproduce only by daughter tubers (Brink, 1980; Kadota, 1987). Additionally, the only dispersal mechanism of Aconitum species reported to date are the falling of the mature seeds near the mother plant due to gravity date (Kadota, 1987). Although A. austrokoreense is designated as an endangered species in Korea, its reproduction, pollination mechanism, seed dispersal, and population dynamics remain elusive. In designing conservation strategies for an endangered species, understanding its genetic structure and variation is of utmost importance.

Microsatellite markers, or simple sequence repeat (SSR) markers, have been widely used in recent population genetics applications as they are highly abundant and polymorphic, co-dominantly inherited, analytically simple, and readily transferable (Weber, 1990; Selkoe and Toonen, 2006). In this study, as a first step in the genetic characterization of A. austrokoreense, we report the development and evaluation of a set of polymorphic microsatellite markers. These results will help evaluate the genetic diversity and facilitate germplasm conservation of this endangered species.

\section{MATERIAL AND METHODS}

Genomic DNA was extracted from silica-gel-dried leaves using the DNeasy Plant Mini Kit (Qiagen, Hilden, Germany) following the manufacturer protocol. The extracted DNA was stored at $-20^{\circ} \mathrm{C}$ and its quality checked by agarose gel electrophoresis $(1 \%)$ and with a spectrophotometer (NanoDrop Technologies, Wilmington, DE, USA). Approximately $10 \mu \mathrm{g}$ of genomic DNA from a single $A$. austrokoreense individual was used to generate the next- 
generation sequencing libraries. Sequencing was carried out on the Roche 454 GS-FLX Titanium platform (1/4 plate) at the National Instrumentation Center for Environmental Management (NICEM, Seoul, Korea). The sequence reads were assembled using Newbler 2.6 (Roche Diagnostics, 454 Life Science, Mannheim, Germany).

To search for microsatellite repeat motifs, di-, tri-, tetra-, penta-, and hexa-nucleotide repeats of more than 4 iterations were searched using the perl program "ssr_finder.pl." The repeats were sorted and a pair of primers flanking each repeat was designed using the Primer3 program (Rozen et al., 2014). Primers were designed using the following criteria: 1) primer size between 18 and $26 \mathrm{bp}$; 2) amplicon size greater than $130 \mathrm{bp}$; and 3) Tm value between 55 and $59^{\circ} \mathrm{C}$. The remaining parameters were left at the default settings. The primer pair with the highest score, according to the Primer3 program, was chosen. In order to fluorescently-label the amplification reactions, a 5'-M13 tail (5'-TGTAAAACGACGGCCAGT-3') was added to the forward primer of each primer pair (Schuelke, 2000).

All PCR amplifications were performed in a final reaction volume of $20 \mu 1$, containing $10 \mathrm{ng}$ genomic DNA, 8 pmol each of the reverse primer and fluorescently labeled (6-FAM, NED, PET, or VIC) M13 primer, 2 pmol of the forward primer, and Premix Taq polymerase (TaKaRa Ex Taq ${ }^{\circledR}$ version 2.0; TaKaRa Bio, Otsu, Japan). PCR amplification conditions were as follows: initial denaturation at $94^{\circ} \mathrm{C}$ for $5 \mathrm{~min} ; 30$ cycles of $30 \mathrm{~s}$ at $94^{\circ} \mathrm{C}, 45 \mathrm{~s}$ at $56^{\circ} \mathrm{C}$, and $45 \mathrm{~s}$ at $72^{\circ} \mathrm{C}$; then 12 cycles of $30 \mathrm{~s}$ at $94^{\circ} \mathrm{C}, 45 \mathrm{~s}$ at $53^{\circ} \mathrm{C}$, and $45 \mathrm{~s}$ at $72^{\circ} \mathrm{C}$; and final extension at $72^{\circ} \mathrm{C}$ for 20 min (Tóth et al., 2000). The size of the PCR products was verified by agarose gel electrophoresis (2\%) and with a spectrophotometer (NanoDrop Technologies). Subsequently, the PCR products were added to Hi-Di ${ }^{\mathrm{TM}}$ formamide with GeneScan ${ }^{\mathrm{TM}} 500$ LIZ size standard (Applied Biosystems, Foster City, CA, USA) and analyzed using an ABI 3730XL DNA Analyzer (Applied Biosystems). The microsatellite profiles were examined using the GeneMarker ${ }^{\circledR}$ program (version 2.4.0; Softgenetics, State College, PA, USA).

To verify the flanking sequences of the microsatellite repeats and to confirm that the intended target fragments were amplified, Sanger sequencing was performed with 8 pmol of forward and reverse primers (unlabeled M13 primer). The amplified products were analyzed using an ABI 3730XL DNA Analyzer and the target sequences were checked for correct amplification using Sequencher (version 5.0; Gene Codes Corp, Ann Arbor, MI, USA).

The proportion of polymorphic loci and the average number of alleles per locus was estimated using the ARLEQUIN software (ver. 3.11) (Excoffier et al., 2005). The observed and expected heterozygosity (HO and HE) and Hardy-Weinberg equilibrium (HWE) were calculated using the same software. MICRO-CHECKER ver. 2.2.3. (Van Oosterhout et al., 2004) was used to check for evidence of null alleles and scoring errors due to stuttering, large allele drop-out, or short allele dominance.

\section{RESULTS AND DISCUSSION}

A total of 58,987 reads (23.02 Mb) were obtained for A. austrokoreense (Table S1). The total number of contigs was 1,094 and the largest contig size was 7,179 bp. In addition, 46,377 singleton sequences for a total read length of $1,865 \mathrm{Mb}$ was obtained. A total of 2,579 microsatellite repeats, containing perfect di-, tri-, tetra-, penta-, and hexanucleotide repeats, were successfully identified using the "ssr_finder.pl" Perl program. Of the dinucleotide repeats, the most frequent dinucleotide in the $A$. austrokoreense genome was the CT repeat (37.68\%), followed by CA (31.55\%), AT (29.46\%), and GC (1.31\%). Of the tri-nucleotide repeats, 
the AAG/AGA/TTC motif was the most frequent (35.87\%), and the GGC repeat motif was the least common. The frequency of tetra-, penta-, and hexanucleotide repeats was very low. These results are consistent with previous findings showing that GA and AAG repeats are the most frequent in vascular plant genomes (Tóth et al., 2000; Kalia et al., 2006). Among the isolated dinucleotide microsatellites, the highest number of repeat-unit iterations was 17 (34 bp), whereas among the trinucleotide microsatellites, the highest number of repeat-unit iterations was 13 (39 bp).

A total of 107 microsatellites, containing the largest number of repeat motifs possible, were chosen to test the amplification efficiency and assess polymorphism. Of these, 39 microsatellites $(36.45 \%)$ produced clear and strongly amplified polymerase chain reaction (PCR) products, while the others produced multiple bands or failed to amplify (as detected by $2 \%$ agarose gel electrophoresis). Sixty-four individuals of A. austrokoreense (Table S2) from two wild populations (Mt. Choejeong in Gyeongsangbuk-do and Ungseokbong in Gyeongsangnam-do) in Korea were used to test the efficiency of the locus-specific primer pairs and to characterize the microsatellite markers.

Nine of the 39 microsatellite primer pairs amplified polymorphic products in the two populations of $A$. austrokoreense (Table 1). The nucleotide sequences of the 9 polymorphic microsatellite markers were deposited in the GenBank database under accession numbers KJ188170-KJ188178 (Table 1). The Mt. Choejeong population yielded 1-4 alleles, with observed heterozygosity $\left(H_{\mathrm{O}}\right)$ values between 0.031 and 0.875 (average of 0.355 ), and expected heterozygosity $\left(H_{\mathrm{E}}\right)$ values between 0.031 and 0.619 (average of 0.352). The Ungseokbong population produced 2-7 alleles, with $H_{\mathrm{O}}$ and $H_{\mathrm{E}}$ values from 0.063 to 0.938 (average of 0.396 ) and from 0.062 to 0.697 (average of 0.390 ), respectively.

Table 1. Set of 9 microsatellite markers developed in Aconitum austrokoreense.

\begin{tabular}{|c|c|c|c|c|c|c|}
\hline Marker name & Repeat motifs & Primer sequence (5'-3') & $\begin{array}{c}\text { Annealing } \\
\left({ }^{\circ} \mathrm{C}\right)\end{array}$ & $\begin{array}{l}\text { Expected } \\
\text { size (bp) }\end{array}$ & $\begin{array}{l}\text { Fluorescent } \\
\text { labels }\end{array}$ & $\begin{array}{c}\text { GenBank } \\
\text { accession No. }\end{array}$ \\
\hline \multirow[t]{2}{*}{ Aau_CA04 } & $\mathrm{TG}_{7}$ & F: M13-AAGATTCCATCCCGATCTTTAT & & & & \\
\hline & & R: ATATCAACGGATCAAACCAAAT & 56 & 240 & NED & KJ188170 \\
\hline \multirow[t]{2}{*}{ Aau_CA05 } & $\mathrm{CA}_{9}$ & F: M13-GGATTTGGTCTGCTCTTCAG & & & & \\
\hline & & R: GTGACACTCATGTGGTCGAA & 56 & 231 & PET & KJ188171 \\
\hline \multirow[t]{2}{*}{ Aau_CA09 } & $(\mathrm{AT})_{2}(\mathrm{CA})_{7}$ & F: M13-GAGTGGGAGATCCACACAAG & & & & \\
\hline & & R: GCTCCAGTTTCCACTTCAAA & 56 & 183 & PET & KJ188172 \\
\hline \multirow[t]{2}{*}{ Aau_ACC01 } & $(\mathrm{ACC})_{10}(\mathrm{TCT})_{2}$ & F: M13-AATTTATAAAACGGATTCGGGT & & & & \\
\hline & & R: ACATTTGGTCATCAAGTTCCTC & 56 & 249 & VIC & KJ188173 \\
\hline \multirow[t]{2}{*}{ Aau_ATA02 } & $\mathrm{TAT}_{7}$ & F: M13-GCTTGAGGGGATTAGAGAGATT & & & & \\
\hline & & R: TCTTTTCCAGTGTTGGAAACTT & 56 & 310 & FAM & KJ188174 \\
\hline \multirow[t]{2}{*}{ Aau_AT37 } & $(\mathrm{AT})_{8} \mathrm{AC}(\mathrm{AT})_{3}$ & F: M13-CCATTGGATGATGGTGTTTT & & & & \\
\hline & & R: CGCAGCAATCACACAAGTAA & 56 & 282 & PET & KJ188175 \\
\hline \multirow[t]{2}{*}{ Aau_TTC04 } & $\mathrm{GAA}_{8}$ & F: M13-TTTAGTTGTTGGTGCCATCC & & & & \\
\hline & & R: TTCATGAACTCTAGCAGGTTACAA & 56 & 179 & FAM & KJ188176 \\
\hline \multirow[t]{2}{*}{ Aau_TTC05 } & $(\mathrm{CT})_{2} \mathrm{TT}(\mathrm{TTC})_{7}$ & F: M13-TCGCAATGGCTACCTCTAAG & & & & \\
\hline & & R: TTGGCTGATAAAGCCTAATTG & 56 & 205 & NED & KJ188177 \\
\hline \multirow[t]{2}{*}{ Aau_ACA03 } & $\mathrm{TGT}_{8}$ & F: M13-GCTCGACGATTCCACAGTA & & & & \\
\hline & & R: TAACTTCTCCGTTCCGCTCT & 56 & 139 & PET & KJ188178 \\
\hline
\end{tabular}

The "expected size" included 18 bp of the M13 tail.

The two populations were tested to determine if they departed from Hardy-Weinberg equilibrium (HWE). In the Mt. Choejeong population, Aau_ACC01, Aau_AT37, Aau_TTC04 $(\mathrm{P}<0.01)$, and Aau_ATA02 loci $(\mathrm{P}<0.05)$ departed from HWE (Table 2). In the Ungseokbong 
population, Aau_CA04, Aau_CA09, Aau_ATA02, Aau_AT37, Aau_TTC05 $(\mathrm{P}<0.01)$, and Aau_TTC04 loci $(\mathrm{P}<0.05)$ departed from HWE (Table $\overline{2})$. The observed departures are probably due to restricted population sizes, genetic drift, vegetative reproduction, and the presence of null alleles. Of the markers, Aau_TTC04 (Mt. Choejeong), Aau_CA04, Aau_ATA02, and Aau_AT37 (Ungseokbong), which departed significantly from HWE, showed homozygote excess by analysis with MICRO-CHECKER. Further, there was no evidence of scoring errors or large allele drop-out in any of the loci. Finally, 9 novel markers satisfied all the criteria (i.e., moderate to high polymorphism, no evidence of null alleles) and are recommended for use in research on the genetic diversity, population structure, gene flow, and mating system of $A$. austrokoreense. In our ongoing research, we are applying these markers to investigate genetic diversity and spatial genetic structure in wild populations of this rare endemic medicinal species. In addition, we expect that these markers will be useful in population genetics, phylogeography, ecology, and conservation efforts of $A$. austrokoreense and its closely related species in the Aconitum genus.

Table 2. Polymorphisms of 9 microsatellite markers developed in Aconitum austrokoreense.

\begin{tabular}{|c|c|c|c|c|c|c|c|c|c|c|c|}
\hline \multirow[t]{2}{*}{ Population } & & \multicolumn{9}{|c|}{ Microsatellite loci } & \multirow[t]{2}{*}{ Mean of all loci } \\
\hline & & Aau_CA04 & Aau_CA05 & Aau_CA09 & Aau_ACC01 & Aau_ATA02 & Aau_AT37 & Aau_TTC04 & Aau_TTC05 & Aau_ACA03 & \\
\hline $\begin{array}{l}\text { Mt. } \\
\text { Choejeong }\end{array}$ & $\begin{array}{l}\text { Size } \\
\text { range }\end{array}$ & $241-243$ & $231-233$ & 184 & $242-251$ & $306-368$ & $288-302$ & $170-179$ & $194-200$ & $137-141$ & \\
\hline \multirow[t]{3}{*}{$(\mathrm{N}=32)$} & $N_{\mathrm{A}}$ & 2 & 2 & 1 & 4 & 3 & 4 & 4 & 3 & 2 & \\
\hline & $H_{\mathrm{O}}$ & 0.031 & 0.188 & - & 0.469 & 0.656 & 0.875 & 0.313 & 0.250 & 0.063 & 0.355 \\
\hline & $H_{\mathrm{E}}$ & 0.031 & 0.173 & - & $0.559^{* *}$ & $0.527^{*}$ & $0.619^{* *}$ & $0.615^{* *}$ & 0.228 & 0.062 & 0.352 \\
\hline \multirow[t]{4}{*}{$\begin{array}{l}\text { Ungseokbong } \\
(\mathrm{N}=32)\end{array}$} & $\begin{array}{l}\text { Size } \\
\text { range }\end{array}$ & $239-245$ & $231-233$ & $184-186$ & $239-242$ & $359-380$ & $292-328$ & $170-173$ & 194-197 & $134-151$ & \\
\hline & $N_{\mathrm{A}}$ & 4 & 2 & 2 & 2 & 4 & 7 & 2 & 3 & 5 & \\
\hline & $H_{\mathrm{o}}$ & 0.063 & 0.094 & 0.488 & 0.063 & 0.219 & 0.531 & 0.313 & 0.938 & 0.656 & 0.396 \\
\hline & $H_{\mathrm{E}}$ & $0.150^{* *}$ & 0.091 & $0.458^{* *}$ & 0.062 & $0.586^{* *}$ & $0.697 * *$ & $0.319^{*}$ & $0.565^{* *}$ & 0.584 & 0.390 \\
\hline
\end{tabular}

$\mathrm{N}$, number of samples; $N_{\mathrm{A}}$, number of alleles; $H_{\mathrm{O}}$, observed heterozygosity; $H_{\mathrm{E}}$, expected heterozygosity. $* \mathrm{P}<0.05$, ${ }^{* *} \mathrm{P}<0.01$ : Significant departure from Hardy-Weinberg equilibrium.

\section{ACKNOWLEDGMENTS}

The authors thank the anonymous reviewers for providing valuable comments on the manuscript. Research supported by a grant (NIBR \#2014-01-003) entitled "The Genetic Evaluation of Important Biological Resources (2014)" from the National Institute of Biological Resources (NIBR) funded by the Ministry of Environment (MOE) of Korea.

\section{Supplementary material}

\section{REFERENCES}

Bessonova IA, Yunusov MS, Kondrat'ev VG and Shreter AI (1987). Alkaloids of Aconitum coreanum. I. Structure of acorine. Chem. Nat. Compd. 23: 573-575.

Bisset NG (1981). Arrow poisons in China (Part II): Aconitum-botany, chemistry, and pharmacology. J. Ethnopharmacol. 4: 247-336.

Bosch M and Waser NM (1999). Effects of local density on pollination and reproduction in Delphinium nuttallianum and Aconitum columbianum (Ranunculaceae). Amer. J. Bot. 86: 871-879.

Brink DE (1980). Reproduction and variation in Aconitum columbianum (Ranunculaceae), with emphasis on California 
populations. Amer. J. Bot. 67: 263-273.

Chung MG and Park C-W (2000). Notes on spatial genetic structure in a hybrid population between Aconitum japonicum subsp. napiforme and A. jaluense (Ranunculaceae). Ann. Bot. Fenn. 37: 243-247.

Excoffier L, Laval G and Schneider S (2005). Arlequin (version 3.0): An integrated software package for population genetics data analysis. Evol. Bioinform. Online 1: 47-50.

He J, Wong K-L, Shaw P-C, Wang H, et al. (2010). Identification of the medicinal plants in Aconitum L. by DNA barcoding technique. Planta Med. 76: 1622-1628.

Kadota Y (1987). A Revision of Aconitum Subgenus Aconitum (Ranunculaceae) of East Asia. Sanwa Shoyaku, Utsunomiya, Tokyo.

Kalia RK, Rai MK, Kalia S, Singh R, et al. (2011). Microsatellite markers: An overview of the recent progress in plants. Euphytica 177: 309-334.

Lee TB (1979). Illustrated Flora of Korea. Hyangmunsa, Seoul. 360-365.

Liang M, Li S, Shen B, Cai J, et al. (2012). Anti-hepatocarcinoma effects of Aconitum coreanum polysaccharides. Carbohydr. Polym. 88: 973-976.

Lyu H-N, Kwak H-Y, Lee D-Y, Kim K-T, et al. (2008). Isolation of flavonoids from processed Aconiti tuber. J. Appl. Biol. Chem. 51: 165-168.

Nautiyal BP, Nautiyal MC, Rawat N and Nautiyal AR (2009). Reproductive biology and breeding system of Aconitum balfourii (Benth) Muk: A high altitude endangered medicinal plant of garhwal Himalaya, India. Res. J. Med. Plant. 3: 61-68.

Oh S and Park C-W (1998). Crossability of the Aconitum jaluense species complex (Ranunculaceae) in Korea. Korean J. Biol. Sci. 2: 435-438.

Park C-W (2007). Aconitum. In: The Genera of Vascular Plants of Korea. Flora of Korea Editorial Committee (Flora of Korea Editorial Committee, eds.). Academy Publishing Co., Seoul, 176-182.

Rastogi S (2011). A review of Aconite (Vatsanabha) usage in Ayurvedic formulations: traditional views and their inferences. Spatula DD. 1: 233-244.

Rozen S and Skaletsky HJ (2003). Primer 3: Bioinformatics Methods and Protocols. In: Methods in Molecular Biology (Krawetz S and Misener S, eds.). Humana Press, Totowa, New Jersey, 365-386. Available at [http://frodo.wi.mit.edu/ cgi-bin/primer3/primer3_www.cgi].

Schuelke M (2000). An economic method for the fluorescent labeling of PCR fragments. Nat. Biotechnol. 18: 233-234.

Selkoe KA and Toonen RJ (2006). Microsatellites for ecologists: a practical guide to using and evaluating microsatellite markers. Ecol. Lett. 9: 615-629.

Suh Y and Kim YJ (2012). Korean Red List of Threatened Species: Mammals, Birds, Reptiles, Amphibians, Fishes and Vascular plants. National Institute of Biological Resources, Incheon, 58.

Suh MH, Koh KS, Ku YB, Kil JH et al. (2004). The conservation strategy for the endangered and reserved plants based on the ecological and genetic characteristics (IV). National Institute of Environmental Research, Incheon, Korea.

Tang Q-Fa, Ye W-C, Liu J-H and Yang C-H (2012). Three new hetisine-type diterpenoid alkaloids from Aconitum coreanum. Phytochem. Lett. 5: 397-400.

Tóth G, Gáspári Z and Jurka J (2000). Microsatellites in different eukaryotic genomes: Survey and analysis. Genome Res. 10: 967-981.

Van Oosterhout C, Hutchinson WF, Wills DPM and Shipley P (2004). MICRO-CHECKER: software for identifying and correcting genotyping errors in microsatellite. Mol. Evol. Notes 4: 535-538.

Weber JL (1990). Informativeness of human (dC-dA)n (dG-dT)n polymorphism. Genomics 7: 524-530.

Yoo BH, Ku YB, Oh HK, Suh MH, et al. (2005). The conservation strategy for the endangered and reserved plants based on the ecological and genetic characteristics (IV). National Institute of Environmental Research, Incheon. 